\title{
Polymer selective laser curing for integrated optical switches
}

\author{
Manuel Gil-Valverde*, Manuel Caño-García, Rodrigo Delgado, Tianyi Zuo, José M. Otón, Xabier Quintana, \\ Morten A. Geday.*m.gilvalverde@upm.es \\ CEMDATIC, ETSI Telecomunicación, Universidad Politécnica de Madrid, Av. Complutense, 30, 28040 Madrid, \\ Spain
}

Received February 15, 2017; accepted March 30, 2017; published March 31, 2017

\begin{abstract}
A simple in-layer electro optical switch has been prepared by selectively curing a photocurable optical polymer with a UV laser. The core of the device is a NOA- 81 multimode waveguide grown by selective laser curing. The cladding is a positive calamitic liquid crystal, which allows tunability and switching of the waveguide by external driving electric signals. The effective refractive index in the guide changes upon switching the liquid crystal. Depending on the geometry, this setup leads to an electrooptical modulator or a switch between two levels of transmitted light.
\end{abstract}

The possibilities offered by polymer-integrated optics are arising. Manufacturing polymer-based devices is less time consuming and reasonable in terms of costs. The refractive indices can be chosen and tuned at will; using core materials having refractive indices close to cladding leads to a low confinement field in the polymer waveguides. This increases the field outside the core, i.e., the evanescent field, allowing for easy modification of overall guiding properties. Tuning the evanescent field is possible by changing the effective refractive index of the cladding, by means of an active electrooptical material such as liquid crystal (LC) [1]. On the other hand, the fabrication process determines the dimensions and shape of the waveguide. Attempts to define simpler processes have led to polymer waveguides as an interesting alternative. Further simplifications mean employing photocurable polymers, since waveguides can be dimensioned and drawn directly using a laser beam for curing.

Several examples of waveguides using polymers have been reported [2][3][4]. In this work, the possibility of preparing active waveguides without photolithographic processes is explored. Our approach shall define the size and shape of the waveguide by photocuring a polymer with a CNC-controlled UV laser. The waveguide will be made tunable by an LC cladding driven by external voltage [5].

The material chosen for the core is the optical adhesive NOA-81 (Norland). The uncured product is a clear liquid with no solvent, which makes it suitable for spin coating processes. The substance is cured under UV light between $320 \mathrm{~nm}$ and $380 \mathrm{~nm}$. It is highly transparent in the range of visible and near infrared light. So it is possible to use it for guiding. Its relatively high refractive index is an advantage when used as a core (Table 1). Fabrication with
NOA-81 does not require special products. It is developed by means of alcohol or acetone. If acetone is used time must be strictly controlled, otherwise the cured polymer can also be removed.

The bottom cladding, i.e., the substrate, is a $700-\mu \mathrm{m}-$ thick glass coated with a $100 \mathrm{~nm}$ layer of indium tin oxide (ITO). This ITO layer will be eventually used as an electrode. The upper cladding is a layer made of MLC 13000 (Merck), a calamitic positive LC with dielectric constants: $\varepsilon_{\mathrm{re}}=30.0$ and $\varepsilon_{\mathrm{ro}}=7.3 @ 1 \mathrm{kHz}$. Its ordinary index is lower than the refractive index of the core (Table 1), while its extraordinary index is higher. Thus, the light will be confined when the LC is off, and the light 'sees' the LC ordinary index. A polyimide layer (PIA-2304D17, Lixon, in DMF 1:3 w/w) is deposited onto the surface to induce an LC alignment direction.

Table 1. Refractive indices at $\lambda=589.3 \mathrm{~nm}$ for the different materials used in the waveguides.

\begin{tabular}{lc}
\hline \multicolumn{1}{c}{ Material } & Refractive index \\
\hline NOA-81 & 1.56 \\
\hline Glass & 1.47 \\
\hline ITO & 1.85 \\
\hline MLC 13000 ordinary $\left(\boldsymbol{n}_{\boldsymbol{o}}\right)$ & 1.49 \\
\hline MLC 13000 extraordinary $\left(\boldsymbol{n}_{\boldsymbol{e}}\right)$ & 1.62 \\
\hline
\end{tabular}

The layer structure in the device was simulated using COMSOL Multiphysics. The refractive indices used for the simulation were taken from Table 1. Different numbers of modes are solved in a section of the guide (Fig. 1). A simplification was performed to reduce the calculation time. The LC volume is split into two parts. The modes are vertically or horizontally polarized. It is considered that vertical oscillations are mainly disturbed by the upper volume while horizontal oscillations are disturbed by the lateral volume. Hence, two different zones were defined for the LC area. A different way that each polarization 'sees' the LC varies depending on the relative orientation of the LC molecules. For instance, when the LC is switched on, the vertical mode detects the extraordinary index above the guide and ordinary index on the sides. So each simulation was performed using two different values of the refractive index. In the absence of voltage, two different orientations are possible (Fig. 2). 


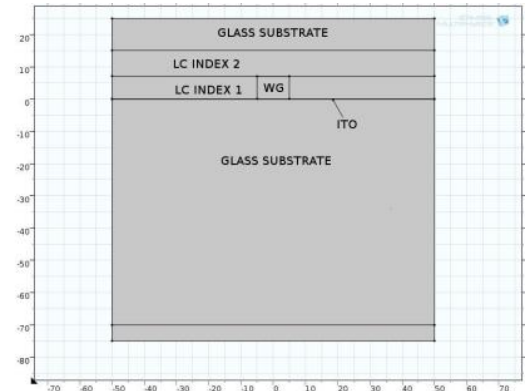

Fig. 1. Structure simulated in COMSOL Multiphysics software. The LC area is split in two layers emulating the way each mode perceives the LC molecules, either laterally or vertically.

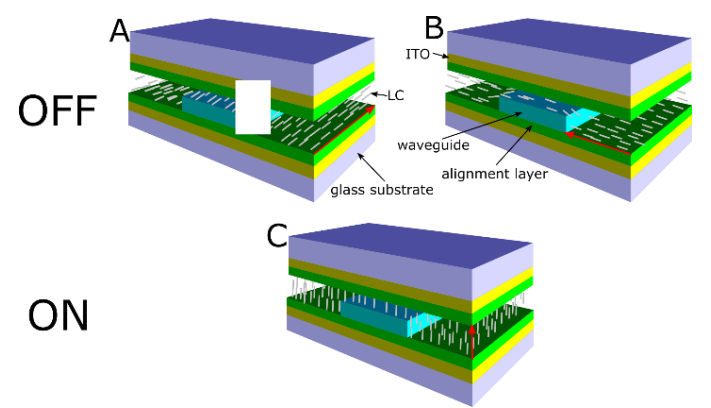

Fig. 2. Layer structure of the device. Two orientations are used when the voltage is off. Both configurations lead to homeotropic orientation upon switching. The red arrows represent the LC orientation direction.

In case $\mathrm{A}$, the $\mathrm{LC}$ indicatrix is parallel to the propagation axis, while in $\mathrm{B}$, the indicatrix is perpendicular. When the voltage is on, the same LC homeotropic orientation is obtained in either case. The simulation results show the power of each mode in a section of the device (Fig. 3). Two modes were calculated for each situation drawn in Fig. 2. Case $\mathrm{A}$ is the best confinement solution with no power guiding outside the guide. In solution B, the first mode is fully confined but the second escapes from the core. In case A the power is lost upon switching. Consequently, orientation A was chosen for fabrication. The LC will switch between A and C.

Glass substrates are cleaned using a plasma bath to enhance adhesion. Next, NOA-81 is deposited by spin coating. The parameters used for spinning the polymer are registered in Table 2 .

Table 2. Spinning parameters for NOA-81 and polyimide.

\begin{tabular}{ccccc}
\hline Material & $\begin{array}{c}\text { Step/Total } \\
\text { steps }\end{array}$ & $\begin{array}{c}\text { Rotation } \\
\text { Speed } \\
\text { (rpm) }\end{array}$ & $\begin{array}{c}\text { Acceleration } \\
\text { (rpm/s) }\end{array}$ & $\begin{array}{c}\text { Total } \\
\text { time (s) }\end{array}$ \\
\hline NOA-81 & $1 / 1$ & 0 to 3000 & 500 & 35 \\
\hline $\begin{array}{c}\text { Poly- } \\
\text { imide }\end{array}$ & $1 / 2$ & 0 to 500 & 100 & 5 \\
\hline
\end{tabular}

Once the coating is deposited but still uncured, the wafer is put into a CNC-controlled laser chamber (SpectraPhysics Laser Processing System LS- $\mu$ PS) delivering 220
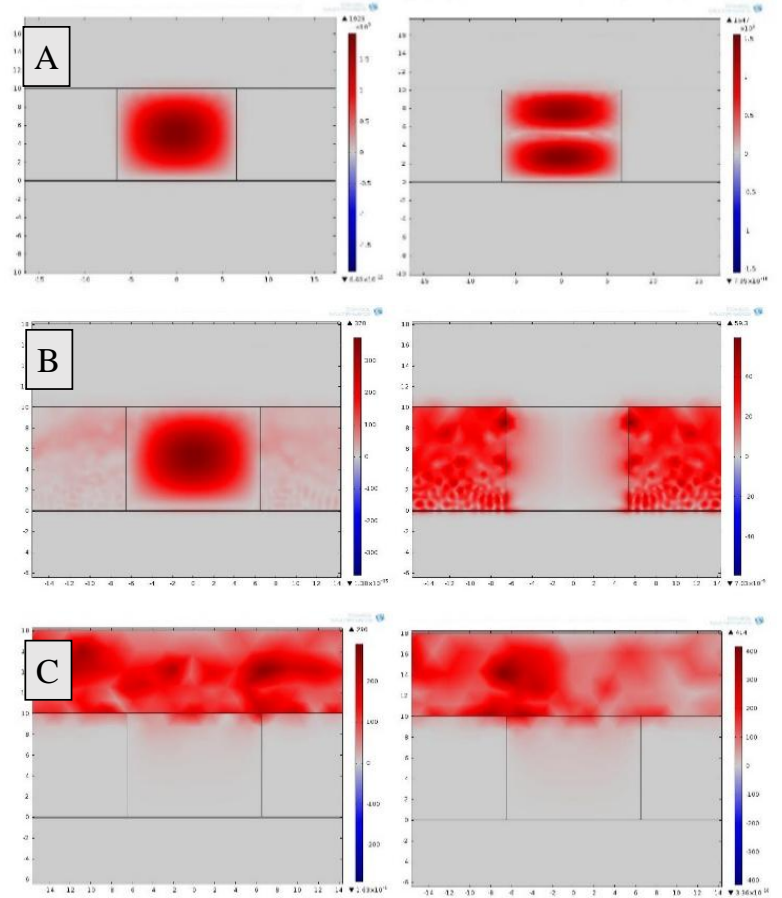

Fig. 3. Power representation for each mode. First modes on the left, second modes on the right. (A) For parallel alignment, first and second modes are mostly confined. (B) For perpendicular alignment, only the first mode is confined with some losses. The second mode is outside the guide. In (C) the switch is on, making the modes leave the core to the

LC upper layer. Only residual guidance is expected in this case.

watts@355nm from a frequency-tripled Nd:YAG, where the selective curing will be performed. The curing beam is focused using a $10 \times$ microscope objective, giving a Gaussian spot of $1.9 \mu \mathrm{m}$. Waveguides were fabricated sweeping the laser times (Fig. 4), from 1 to 900 sweeps. Depending on the power, several sweeps are usually required to fully cure the material. Different sweeps also give different waveguide sizes.

The speed of a laser sweep was set to $10 \mathrm{~mm} / \mathrm{s}$. With this setup, the laser output power was attenuated down to $0.185 \mathrm{~mW}$, so that the energy density $\left(3.5 \mathrm{~J} / \mathrm{cm}^{2}\right)$ was slightly over the recommended density for NOA-81 curing $\left(2.0 \mathrm{~J} / \mathrm{cm}^{2}\right)$ After curing, the guides are washed out in acetone for $40 \mathrm{~s}$ to remove the uncured NOA-81 [6].

The guide fabrication starts with depositing a polyimide layer by spin coating onto the ITO side of the glass substrate. The spinning parameters can be found in Table 2. The polyimide is cured at $180^{\circ} \mathrm{C}$ for 1 hour and rubbed to set the orientation of the LC [7]. Depositing the polyimide before depositing NOA-81 prevents damages on the waveguide when rubbing. NOA-81 is spin-coated and cured as described above. The top glass substrate is prepared by spinning and rubbing the polyimide as above. A mixture of NOA-81 and $18 \mu \mathrm{m}$ microspacers is used to seal the substrates. The sealed cell (Figure 4) must be left under UV light for one or two minutes. The LC is 
introduced by capillarity. Finally, electrical connections are attached to the ITO electrodes.
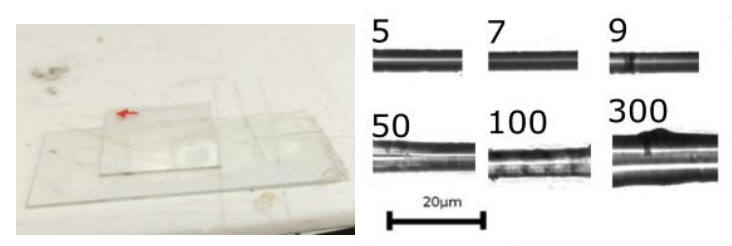

Fig. 4. Left, image of the switch. The LC pretilt is compensated by antiparallel alignment. Right, microscope images of waveguides selectively cured using different numbers of laser sweeps.

A standard $9 \mu \mathrm{m}$-core fiber connected to the switching core was used for light coupling. Index matching was achieved with a drop of NOA-81.

Different numbers of laser sweeps in the fabrication lead to different waveguide shapes. Hence, a collection of different shape waveguides was obtained. Then their height and width were measured using a profilometer. The recorded data are shown in Figure 5. Increasing the number of sweeps increases both the width and height of the waveguide. The height rises more slowly than the width, due to the developing process. More sweeps make the polymer harder, so it is more difficult to be removed by acetone. The width is more tuneable because increasing power allows the contiguous NOA-81 to cure and be added to the guide.

A valid range of good quality guides from 20 to 50 sweeps was chosen. The final microscopic inspection was performed to reject guides with imperfections. Guides fabricated with insufficient energy can peel off the substrate. If the dose is excessively high (more than 90 sweeps), inhomogeneities show up.

The final devices are able to switch between two states (Fig. 6). Regarding the core structure, the waveguide tip shows a larger width than the waveguide. This is an artifact due to the laser stop after sweeping along the waveguide track. Several hardware and software solutions are currently being tested to overcome this problem.

Two different intensity levels are clearly seen upon switching. As expected from the simulations, the power output is not zero in either case.

In conclusions, an optical switch based on a photocured polymer core and LC cladding has been created using a CNC-controlled UV laser. First feasible devices were made for multimode guiding in visible light.

Making waveguides by selective laser curing seems to be a fast and inexpensive method for integrated polymer devices.

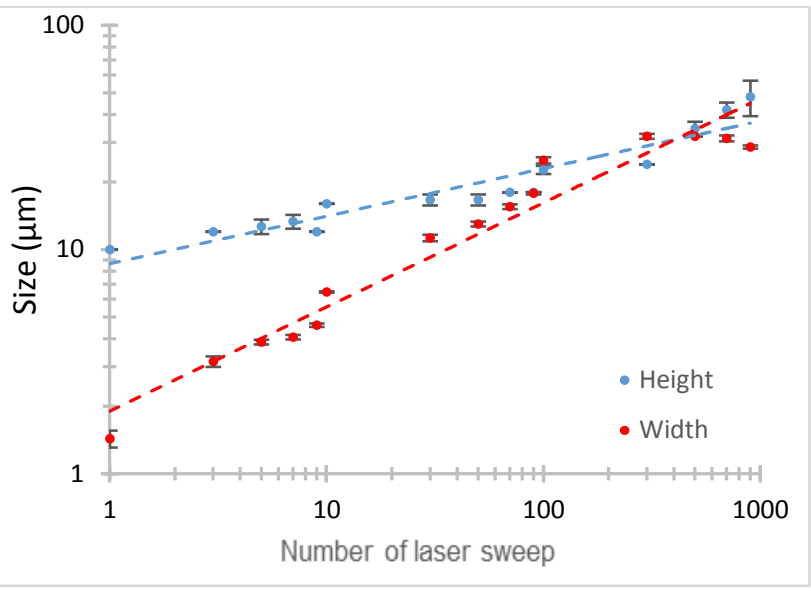

Fig. 5. Height and width of different waveguides made by selective curing at many different laser sweeps.

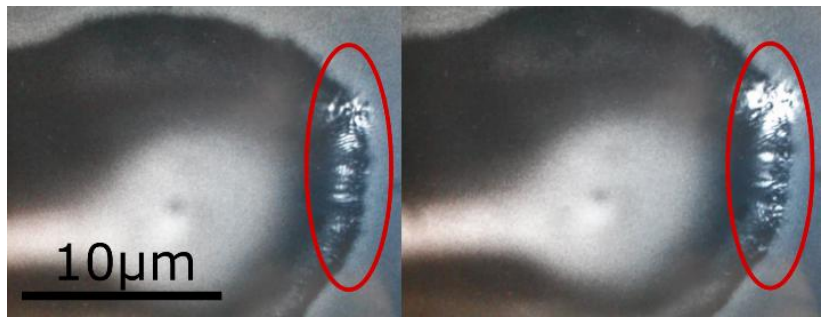

Fig. 6. Tip of the polymer waveguide guiding light. In both cases light is detected at the end of the guide; light intensity differs from the switchedon state (left) to the switched-off state (right).

\section{References}

[1] T. Ako, A. Hope, T. Nguyen, A. Mitchell, W. Bogaerts, K. Neyts, and J. Beeckman, Opt. Express 23, 2846 (2015).

[2] K. Kruse, C. Middlebrook, Photon. Nanostruct. - Fundamentals and Appl. 13, 66 (2015).

[3] A. Günther, A.B. Petermann, M. Rezem, M. Rahlves, M. Wollweber, and B. Roth, European Conf. Lasers and ElectroOptics - European Quantum Electronics Conference, Munich, Germany (2015).

[4] C. Florian, S. Piazza, A. Diaspro, P. Serra, M. Duocastella, ACS Appl. Mater. Interfaces, 8(27), 17028 (2016).

[5] F. Costache, M. Blasl, Opt. Photonik 6, 29 (2011).

[6] M. Caño-Garcia, R. Delgado, T. Zuo, M.A. Geday, X. Quintana, Jose M. Otón, $16^{\text {th }}$ OLC Topical Meeting on the Optics of Liquid Crystals, Sopot, Poland (2015).

[7] S. Ishihara, H. Wakemoto, K. Nakazima, Y. Matsuo, Liq. Cryst. 4(6), 669 (1989). 